\title{
Avaliação nutricional e comportamento alimentar entre mulheres em tratamento de câncer de mama
}

Nutritional assessment and eating behavior among women undergoing breast cancer treatment

Evaluación nutricional y conducta alimentaria en mujeres sometidas a tratamiento contra el cáncer de mama

Recebido: 28/09/2021 | Revisado: 05/10/2021 | Aceito: 12/10/2021 | Publicado: 14/10/2021

\author{
Mauro Celso de Souza \\ ORCID: https://orcid.org/0000-0003-4988-2879 \\ Universidade do Oeste de Santa Catarina, Brasil \\ E-mail: mauroosouzaa@ hotmail.com \\ Patricia Karine Zastrow \\ ORCID: https://orcid.org/0000-0002-0473-9385 \\ Universidade do Oeste de Santa Catarina, Brasil \\ E-mail: patriciakaarine@gmail.com \\ Marceli Pitt Coser \\ ORCID: https://orcid.org/0000-0003-0430-6392 \\ Universidade do Oeste de Santa Catarina, Brasil \\ E-mail: marceli.coser@unoesc.edu.br
}

\begin{abstract}
Resumo
Objetivo: Avaliar o comportamento alimentar, influências e percepções de mulheres após o diagnóstico de câncer de mama e analisar o estado nutricional das mesmas durante o tratamento antineoplásico. Metodologia: Pesquisa transversal, descritiva, de campo, com abordagem quantitativa. Foram coletados dados em dois centros da Rede Feminina de Combate ao Câncer e em um hospital oncológico, ambos em Santa Catarina, com 36 pacientes, por meio de entrevista individual. Resultados: A maioria das participantes apresentou peso acima do adequado e risco para doenças metabólicas e cardiovasculares. Modificações nos hábitos alimentares foram relatados, bem como, instruções sobre condutas alimentares de profissionais da saúde. Entretanto, as maiores motivações para as mudanças ocorridas foram através de amigos e/ou familiares e internet. $\mathrm{O}$ tratamento antineoplásico mais prevalente foi o quimioterápico, acompanhado de sintomas como fraqueza, náusea e inapetência. Percebeu-se a diminuição e/ou exclusão de alimentos açucarados/doces, gorduras, carnes vermelhas, refrigerantes e bebidas alcoólicas, e inclusão de frutas, hortaliças, chás, sucos naturais e água na dieta após o diagnóstico. Conclusão: Evidenciou-se que o estado nutricional preditivo às doenças metabólicas e cardíacas prevaleceu. Houve susceptibilidade às mudanças alimentares, mas pouco influenciadas pelos profissionais que as acompanham no tratamento, inferindo na necessidade de novas estratégias de exposição de saberes, e a constante necessidade de informação.
\end{abstract}

Palavras-chave: Neoplasias da mama; Oncologia; Dietética; Comportamento alimentar.

\begin{abstract}
Objective: Evaluate the eating behavior, influences and perceptions of women after the diagnosis of breast cancer and analyze their nutritional status during cancer treatment. Methodology: Cross-sectional, descriptive, field research with a quantitative approach. Data were collected in two centers from the Women's Network for Combat to Cancer and in an Oncology Hospital, both in Santa Catarina, with 36 patients, through individual interviews and structured questionnaires developed by the authors. Results: Most participants were overweight and were at risk for metabolic and cardiovascular diseases. Changes in eating habits were reported, as well as instructions on eating behaviors from health professionals. However, the biggest motivations for the changes that occurred were through friends and/or family and the internet. The most prevalent antineoplastic treatment was chemotherapy, accompanied by symptoms such as weakness, nausea and inappetence. A reduction and/or exclusion of sugary/sweet foods, fats, red meat, soft drinks and alcoholic beverages was noticed, and the inclusion of fruits, vegetables, teas, natural juices and water in the diet after diagnosis. Conclusion: It was evident that the predictive nutritional status of metabolic and cardiac diseases prevailed. There was susceptibility to dietary changes, but little influenced by the professionals who accompany them in the treatment, inferring the need for new knowledge exposure strategies, and the constant need for information.
\end{abstract}

Keywords: Breast neoplasms; Oncology; Dietetics; Eating behavior.

\section{Resumen}

Objetivo: Evaluar la conducta alimentaria, las influencias y las percepciones de las mujeres tras el diagnóstico de cáncer de mama y analizar su estado nutricional durante el tratamiento del cáncer. Metodología: Investigación 
transversal, descriptiva, de campo, con enfoque cuantitativo. Los datos fueron recolectados en dos centros de la Red de Mujeres de Combate al Cáncer y en un hospital de oncología, ambos en Santa Catarina, con 36 pacientes, a través de entrevistas individuales. Resultados: La mayoría de las participantes presentaba sobrepeso y riesgo de enfermedades metabólicas y cardiovasculares. Se informaron cambios en los hábitos alimenticios, así como instrucciones sobre conductas alimentarias de los profesionales de la salud. Sin embargo, las mayores motivaciones para los cambios que ocurrieron fueron a través de amigos y/o familiares e Internet. El tratamiento antineoplásico más prevalente fue la quimioterapia, acompañada de síntomas como debilidad, náuseas e inapetencia. Se notó reducción y/o exclusión de alimentos azucarados/dulces, grasas, carnes rojas, refrescos y bebidas alcohólicas, y la inclusión de frutas, verduras, tés, jugos naturales y agua en la dieta luego del diagnóstico. Conclusión: se evidenció que prevaleció el estado nutricional predictivo de enfermedades metabólicas y cardíacas. Hubo susceptibilidad a cambios en la dieta, pero poco influenciada por los profesionales que los acompañan en el tratamiento, infiriendo la necesidad de nuevas estrategias de exposición de conocimientos y la necesidad constante de información.

Palabras clave: Neoplasias mamarias; Oncología; Dietética; Comportamiento alimenticio.

\section{Introdução}

Descrito como um conjunto de mais de cem doenças, o câncer (CA) possui como característica primária o crescimento celular desordenado, desencadeando a perda do controle de divisão das células, podendo adentrar tecidos e órgãos vizinhos (Instituto Nacional do Câncer José Alencar Gomes da Silva [INCA], 2020a). No Brasil, o tipo de CA mais incidente entre as mulheres é o de mama, onde se estima o diagnóstico de 66.280 novos casos para cada ano do triênio 2020 e 2022 (INCA, 2019a).

Dentre os fatores contribuintes para o desencadeamento do CA de mama, o envelhecimento (pessoas com mais de 50 anos) é o que possui maior impacto, seguido por causas ambientais e comportamentais, como sobrepeso e obesidade após a menopausa, sedentarismo, exposições frequentes a radiações ionizantes e consumo de bebidas alcoólicas. Fatores da história hormonal, reprodutiva, genética e hereditárias também são predisponentes. Cerca de $30 \%$ dos casos de CA de mama poderiam ser evitados através da alimentação saudável, manutenção do peso corporal adequado, prática de atividade física, amamentação e o não consumo de bebidas alcoólicas (INCA, 2020b).

Não há doença que não tenha algum de seus componentes determinados, favorecidos ou até de algum modo, influenciados pelas condições da nutrição. A doença neoplásica maligna pode ter, em pelo menos 30\% das vezes, com prevalência crescente, origem em maus hábitos alimentares, obesidade e sedentarismo (Sawaya et al., 2018).

$\mathrm{O}$ estado nutricional dos pacientes com $\mathrm{CA}$ de mama pode ser afetado devido às modalidades de tratamento que podem causar efeitos colaterais (Palmieri et al., 2013). As necessidades nutricionais são modificadas antes, durante e após o tratamento de tumores (Patterson et al., 2003). A terapia nutricional atua com o objetivo de reduzir os efeitos colaterais, garantir um aporte necessário e adequado de nutrientes, manter a atividade do sistema imunológico, reduzir o risco de complicações oriundas dos tratamentos e melhorar a qualidade de vida. A terapia nutricional pode alterar o processo carcinogênico independente do estágio em que se encontra, incluindo metabolismo, defesa celular e crescimento tumoral. A nutrição é afetada tanto pelo CA quanto pelo tipo de tratamento realizado (Mahan \& Raymond, 2018).

Hodiernamente, a população pode encontrar uma ampla quantidade de dados e informações sobre alimentação e saúde. Muitas vezes, os pacientes recebem informações que acabam gerando dúvidas e conflitos, que quando associados a tabus, aversões, preferências e crenças, podem alterar o comportamento alimentar e afetar seu estado nutricional (Cicco et al., 2006).

Sendo assim, o objetivo deste trabalho foi avaliar o comportamento alimentar, influências e percepções de mulheres pacientes de um hospital oncológico e/ou frequentadoras de dois centros da Rede Feminina de Combate ao Câncer (RFCC), após o diagnóstico de CA de mama, e analisar o estado nutricional durante o tratamento antineoplásico. 


\section{Metodologia}

Esse estudo se caracteriza como uma pesquisa transversal e descritiva, na qual "descrever os fatos e fenômenos de determinada realidade" (Triviños, 1987). De campo, onde "caracteriza-se pelas investigações em que, além da pesquisa bibliográfica e/ou documental, se realiza coleta de dados junto a pessoas, com o recurso de diferentes tipos de pesquisa" (Fonseca, 2002). Com abordagem quantitativa, que para Pereira et al. (2018), "faz-se a coleta de dados quantitativos ou numéricos por meio do uso de medições de grandezas e obtém-se por meio da metrologia, números com suas respectivas unidades".

A coleta de dados foi realizada em dois momentos. O primeiro entre setembro e outubro de 2019 em dois centros da RFCC de duas cidades distintas. E o segundo, no mês de fevereiro de 2021 nas dependências de um hospital oncológico. Tanto as RFCC quanto o hospital oncológico se localizam na região Meio Oeste de Santa Catarina.

Participaram da pesquisa mulheres acima de dezoito anos de idade, em tratamento de CA de mama, que frequentaram os locais de estudo nos dias das coletas de dados, que aceitaram participar da pesquisa mediante assinatura do Termo de Consentimento Livre e Esclarecido (TCLE), e que souberam responder todas as questões do questionário. Não participaram da pesquisa aquelas com o diagnóstico da doença e que não iniciaram ou já encerraram a terapia oncológica. A população do estudo contemplou onze mulheres frequentadoras das RFCC e vinte e cinco do hospital oncológico. O cálculo amostral levou em consideração o erro de $10 \%$ e nível de confiança de $95 \%$, totalizando trinta e seis participantes.

Por meio de entrevista individual foi realizada a coleta de dados. Primariamente, foram explicados os objetivos, riscos, benefícios e outras informações pertinentes às interessadas. Posteriormente, foi aplicado um questionário estruturado pelos autores, contendo quatro perguntas fechadas sobre dados socioeconômicos, seis perguntas abertas e com alternativas sobre os hábitos alimentares, e verificado seis dados para avaliação antropométrica. Para análise da frequência alimentar foi aplicado um questionário adaptado de Viebig e Valero (2004), que investigou a ingesta de nove grupos alimentares.

O peso corporal total foi obtido através de uma balança da marca Wiso®, modelo W939, com capacidade para 150 quilogramas (kg) e precisão de 100 gramas (g). As participantes foram orientadas a manter as roupas leves, retirando casacos, adornos e calçados. A estatura foi verificada por meio de estadiômetro portátil da marca Cardiomed ${ }^{\circledR}$ com precisão de 1 milímetro (mm), sendo orientadas a permanecerem eretas, com os pés unidos e paralelos. Para as medidas de circunferências, foi utilizada uma fita métrica não elástica da marca Cescorf®, com precisão de $0,1 \mathrm{~mm}$. Foi verificado e classificado o risco cardiovascular pela relação cintura-quadril (RCQ), obtida pela divisão dos perímetros de cintura em centímetros (cm) e quadril (cm) (World Health Organization [WHO], 1997). Foi obtido e avaliado o Índice de Massa Corporal (IMC) (WHO). A medida da circunferência do braço foi tomada preferencialmente no braço direito, e seguiu-se as recomendações metodológicas (Cuppari, 2005). Através desse dado, foi avaliado a porcentagem de adequação, e então a classificação do estado nutricional (Frisancho, 1981). Foi utilizada a medida da circunferência do abdômen em $\mathrm{cm}$ para determinar a presença de risco para complicações metabólicas (WHO).

Os dados coletados foram compilados e expostos em figuras, tabelas, bem como, de forma descritiva quando pertinente. Para análise e estatística foram utilizadas as ferramentas do Microsoft@ Word, Excel versão 2016 e GraphPadPrism® 7.0. Esta pesquisa foi aprovada pelo Comitê de Ética em Pesquisa da Universidade do Oeste de Santa Catarina (UNOESC) sob CAAE n 18822419.1.0000.5367 e parecer 3.981.527.

\section{Resultados}

Do total de mulheres com CA de mama em tratamento avaliadas, a maioria eram casadas, aposentadas ou afastadas, possuem ensino fundamental incompleto ou ensino médio completo e uma renda de um a dois salários mínimos. A média de idade foi de 53,11 $\pm 9,84$ anos. O perfil sociodemográfico completo pode ser observado na tabela 1 . 
Research, Society and Development, v. 10, n. 13, e316101321169, 2021

(CC BY 4.0) | ISSN 2525-3409 | DOI: http://dx.doi.org/10.33448/rsd-v10i13.21169

Tabela 1. Perfil sociodemográfico de mulheres em tratamento de câncer de mama.

\begin{tabular}{|c|c|c|}
\hline VARIÁVEIS & Número (n) & Porcentagem (\%) \\
\hline \multicolumn{3}{|l|}{ Local da Pesquisa } \\
\hline Rede de Combate ao câncer 1 & 3 & 8,3 \\
\hline Rede de Combate ao câncer 2 & 8 & 22,2 \\
\hline Hospital Oncológico & 25 & 69,5 \\
\hline \multicolumn{3}{|l|}{ Estado civil } \\
\hline Solteira & 3 & 8,3 \\
\hline Casada & 20 & 55,6 \\
\hline Divorciada & 7 & 19,4 \\
\hline Viúva & 6 & 16,7 \\
\hline \multicolumn{3}{|l|}{ Ocupação atual } \\
\hline Trabalhando & 2 & 5,6 \\
\hline Aposentada & 18 & 50,0 \\
\hline Afastada & 16 & 44,4 \\
\hline \multicolumn{3}{|l|}{ Nível de escolaridade } \\
\hline Não alfabetizado & 0 & 0 \\
\hline Ensino fundamental incompleto & 11 & 30,6 \\
\hline Ensino fundamental completo & 4 & 11,1 \\
\hline Ensino médio incompleto & 4 & 11,1 \\
\hline Ensino médio completo & 12 & 33,3 \\
\hline Ensino superior incompleto & 1 & 2,8 \\
\hline Ensino superior completo & 4 & 11,1 \\
\hline \multicolumn{3}{|l|}{ Renda } \\
\hline 1 a 2 salários & 32 & 88,9 \\
\hline 3 a 5 salários & 4 & 11,1 \\
\hline 6 salários ou mais & 0 & 0 \\
\hline Outro & 0 & 0 \\
\hline
\end{tabular}

Fonte: dados da pesquisa.

Foi analisado o perfil antropométrico nutricional das mulheres em tratamento de CA de mama. Na tabela 2 é possível correlacionar a média e o desvio padrão dos dados coletados em seus âmbitos. 
Tabela 2. Características nutricionais antropométricas entre mulheres em tratamento de câncer de mama.

\begin{tabular}{lc}
\hline \multicolumn{1}{c}{ Variáveis } & Total Média $\pm \mathbf{D P}(\mathbf{n}=\mathbf{3 6})$ \\
\hline Massa corporal $(\mathrm{kg})$ & $70,9 \pm 12,1$ \\
Estatura $(\mathrm{cm})$ & $158 \pm 6,9$ \\
$\mathrm{IMC}\left(\mathrm{kg} / \mathrm{m}^{2}\right)$ & $28,4 \pm 5,4$ \\
Circunferência do braço $(\mathrm{cm})$ & $32,2 \pm 4,2$ \\
Circunferência da Cintura $(\mathrm{cm})$ & $92,2 \pm 12,0$ \\
Circunferência do Abdômen $(\mathrm{cm})$ & $98,0 \pm 14,0$ \\
Circunferência do Quadril $(\mathrm{cm})$ & $105,5 \pm 11,5$ \\
RCQ & $0,93 \pm 0,06$ \\
\hline
\end{tabular}

DP: desvio padrão. n: número. kg: quilogramas. m. metros. IMC: índice de massa corporal. $\mathbf{k g} / \mathbf{m}^{2}$ : quilogramas por metro quadrado. $\mathbf{c m}$ : centímetros. RCQ: relação cintura quadril.

Fonte: dados da pesquisa.

Quanto a classificação antropométrica, conforme o IMC (figura 1A), pode-se observar que a maioria das entrevistadas está acima do recomendado, entre sobrepeso ou obesidade grau I, e apenas $27,8 \%$ se encontra em eutrofia. Pela circunferência do braço (figura 1B), metade das entrevistadas estão eutróficas, seguido de obesidade ou sobrepeso. Pela circunferência abdominal (figura 1C), que possui como marcador o risco para complicações metabólicas, observa-se que 80,6\% apresenta risco muito aumentado. Conforme a classificação da relação cintura/quadril (figura 1D), 66,7\% demonstra risco às doenças cardiovasculares.

Figura 1. Classificação do estado nutricional conforme diferentes parâmetros antropométricos entre mulheres em tratamento de câncer de mama.
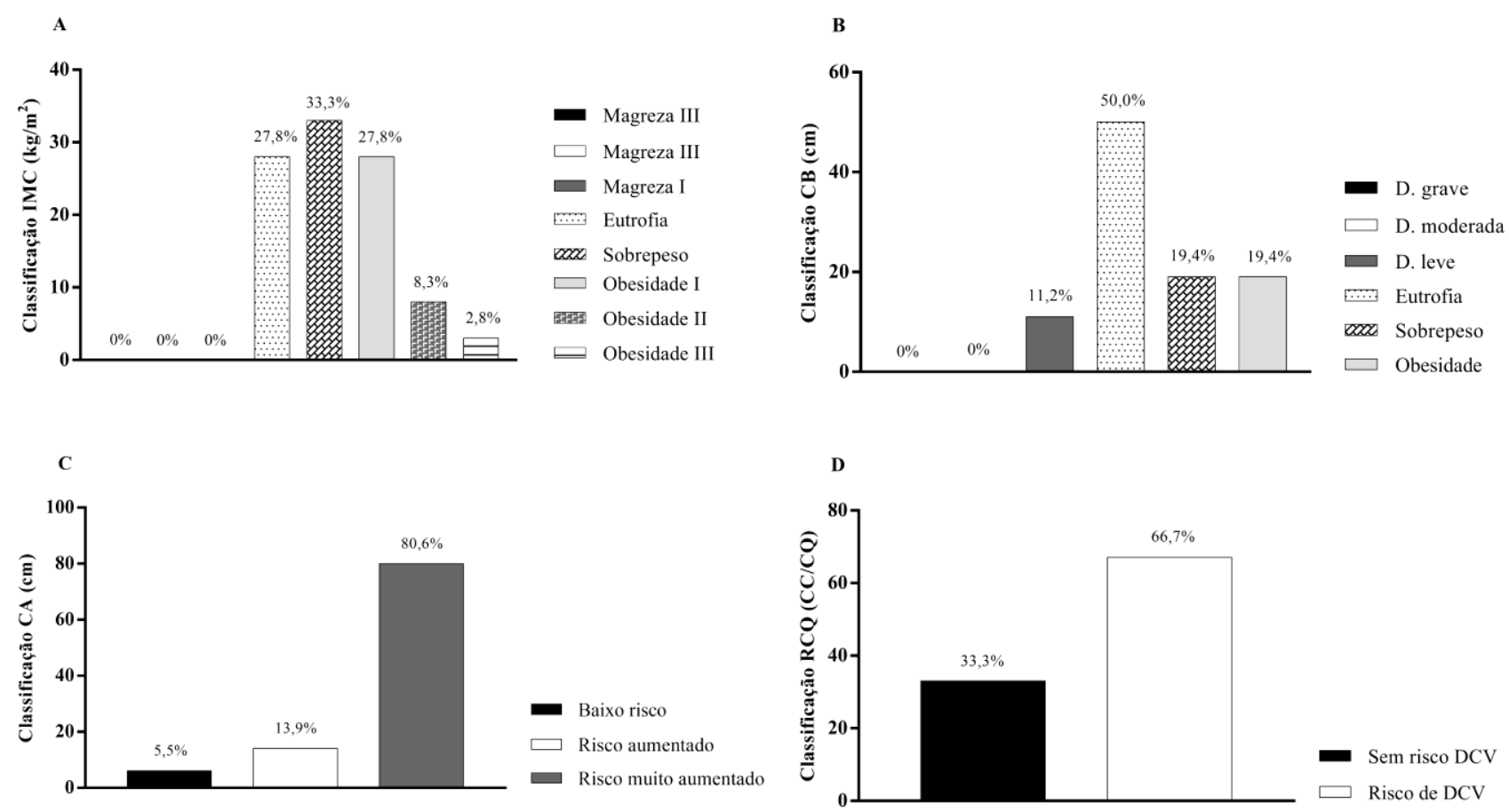

IMC: índice de massa corporal. $\mathbf{K g} / \mathbf{m}^{2}$ : quilogramas por metro quadrado. $\mathbf{C B}$ : circunferência do braço. Cm: centímetros. D: desnutrição. CA: circunferência do abdômen. RCQ: relação cintura quadril. CC/CQ: circunferência da cintura dividida pela circunferência do quadril. DCV: doença cardiovascular. (A) Classificação conforme IMC. (B) Classificação conforme CB. (C) Risco de complicações metabólicas conforme CA. (D) Risco de DCV pela RCQ. Valores expressos em porcentagem (\%). Fonte: dados da pesquisa. 
No que se refere ao tratamento oncológico realizado, $88,9 \%$ das mulheres recebem tratamento quimioterápico (figura 2A). Ao analisar a sintomatologia, é possível observar que $83,33 \%$ relacionam algum tipo de sintoma ao decorrer do tratamento, sendo fraqueza/fadiga, náuseas e inapetência os mais apontados, seguidos de vômitos e diarreia. Apenas 16,67\% não sentiram qualquer sintoma durante o tratamento (figura 2B).

Figura 2. Tipo de terapia oncológica e sintomas apresentados por mulheres em tratamento contra o câncer de mama.
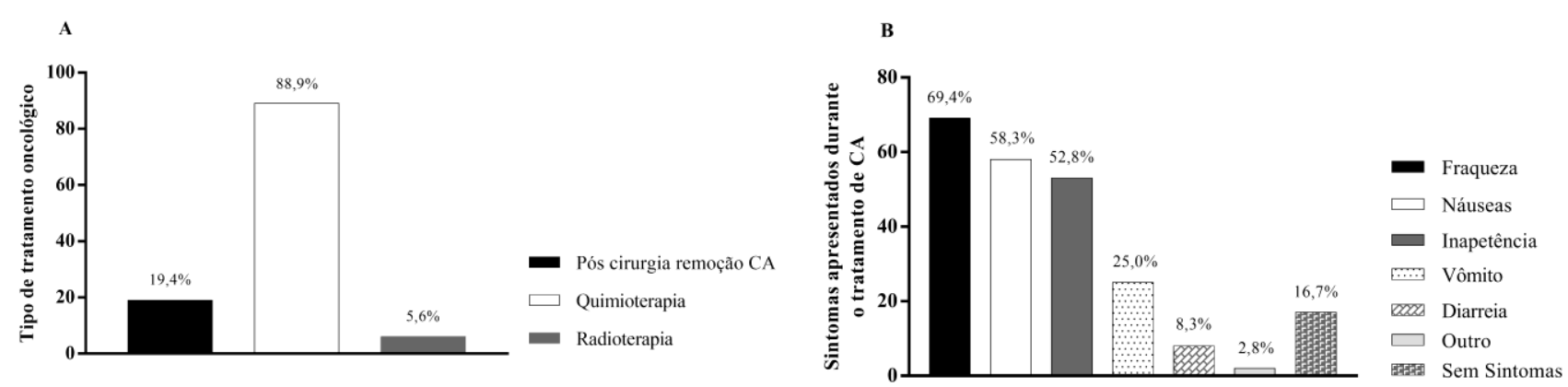

CA: câncer. (A) Tipo de tratamento oncológico realizado. (B) Sintomas apresentados durante o tratamento contra o câncer. Valores expressos em porcentagem (\%).

Fonte: dados da pesquisa.

Ao correlacionar os sintomas com o tipo de tratamento oncológico, nota-se que durante a quimioterapia houve uma maior diversidade de sintomas indesejados do que nos demais tipos de terapia. Na tabela 3 é possível observar todos os sintomas apresentados em cada tipo de tratamento entre as mulheres estudadas.

Tabela 3. Relação dos sintomas e o tipo de tratamento entre mulheres em tratamento de câncer de mama.

\begin{tabular}{ccccc}
\hline & \multicolumn{5}{c}{ TRATAMENTO } \\
\cline { 2 - 5 } SINTOMAS & $\begin{array}{c}\text { Pós cirurgia } \\
11,1 \%(\mathrm{n}=4)\end{array}$ & $\begin{array}{c}\text { Quimioterapia } \\
80,6 \%(\mathrm{n}=29)\end{array}$ & $\begin{array}{c}\text { Pós cirurgia e } \\
\text { quimioterapia } \\
2,8 \%(\mathrm{n}=1)\end{array}$ & $\begin{array}{c}\text { Pós cirurgia, } \\
\text { quimioterapia } \\
\text { e radioterapia } \\
5,6 \%(\mathrm{n}=2)\end{array}$ \\
\hline Náusea & $66,7 \%(\mathrm{n}=3)$ & $51,7 \%(\mathrm{n}=15)$ & - & $100 \%(\mathrm{n}=2)$ \\
Vômito & $25,0 \%(\mathrm{n}=1)$ & $24,1 \%(\mathrm{n}=7)$ & - & $50,0 \%(\mathrm{n}=1)$ \\
Inapetência & $50,0 \%(\mathrm{n}=2)$ & $51,7 \%(\mathrm{n}=15)$ & - & $100 \%(\mathrm{n}=2)$ \\
Diarreia & $25,0 \%(\mathrm{n}=1)$ & $3,4 \%(\mathrm{n}=1)$ & - & $50,0 \%(\mathrm{n}=1)$ \\
Fraqueza/Fadiga & $50,0 \%(\mathrm{n}=2)$ & $72,4 \%(\mathrm{n}=21)$ & - & $100 \%(\mathrm{n}=2)$ \\
Outro & - & $3,4 \%(\mathrm{n}=1)$ & - & - \\
Nenhum & $25,0 \%(\mathrm{n}=1)$ & $13,8 \%(\mathrm{n}=4)$ & $100 \%(\mathrm{n}=1)$ & - \\
\hline
\end{tabular}

$\%$ : porcentagem. N: número.

Fonte: dados da pesquisa.

Sobre acreditar na influência da alimentação no tratamento do CA de mama, 83,3\% das participantes julgaram ter impacto (figura 3A). Ao analisar a (s) possível (eis) mudança (s) de hábito (s) alimentar (es) após o diagnóstico e/ou tratamento do CA, é possível observar que mais da metade das mulheres aumentou ou diminuiu algum alimento da sua dieta e, em relação as bebidas, $77,1 \%$ incluiu alguma na sua rotina e 54,1\% excluiu alguma bebida (figura 3B). Dentre aquelas que diminuíram/excluíram um ou mais alimentos, é possível verificar que essa mudança foi principalmente em relação ao consumo de açúcar e/ou alimentos doces da dieta, alimentos gordurosos/frituras e carnes vermelhas, seguido de demais alimentos (figura 
3C). Entre aquelas que aumentaram/incluíram alimentos, se observa que a mudança foi majoritariamente em relação ao consumo de hortaliças e frutas em uma proporção de 65,4\% para ambos (figura 3D). Expressivamente, o refrigerante (80\%) foi a bebida mais restringida da dieta após o diagnóstico do CA entre aquelas que relataram ter diminuído/excluído bebida, seguido por bebidas alcoólicas, café e chimarrão (figura 3E). Já sobre o aumento/inclusão, o chá se destacou, sendo relatado consumo por 77,8\% das participantes, seguido por sucos naturais, água, leite e água de coco (Figura 3F).

Figura 3. Análise da credibilidade sobre o papel da alimentação no tratamento oncológico, e as mudanças do comportamento alimentar após diagnóstico da doença entre mulheres com câncer de mama.
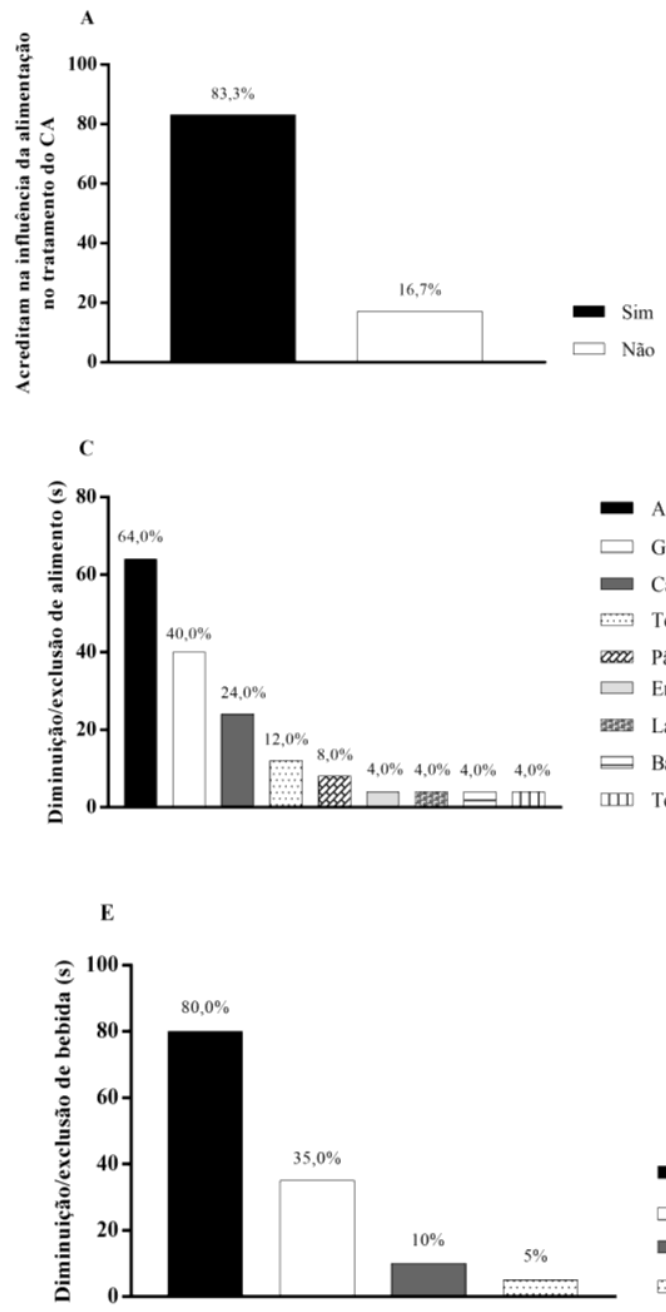
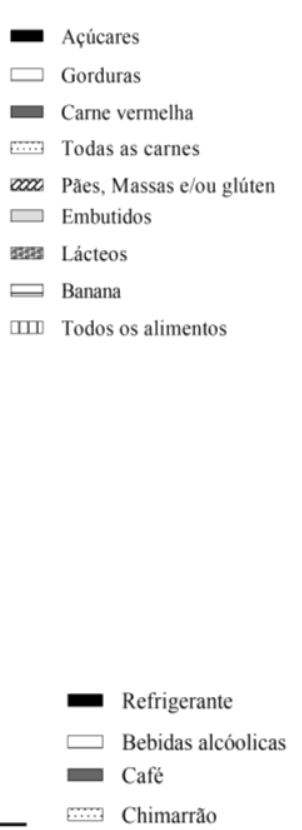
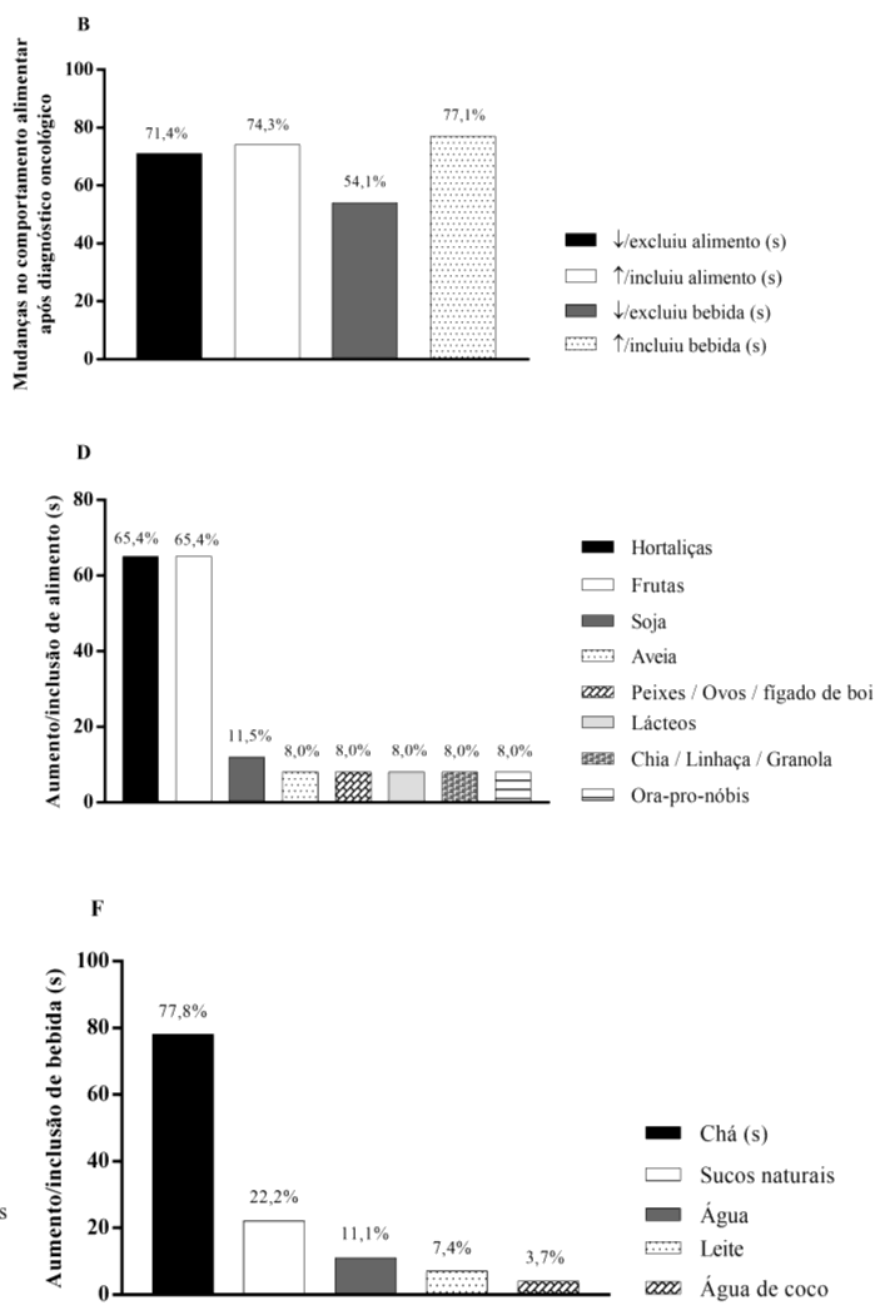

CA: câncer. $\downarrow$ : diminuiu. $\uparrow:$ aumentou. (A) Credibilidade sobre a influência da alimentação no tratamento de câncer. (B) Mudanças comportamentais alimentares após diagnóstico da doença. (C) Alimentos diminuídos/excluídos da dieta após diagnóstico da doença. (D) Alimentos aumentados/incluídos na dieta após diagnóstico da doença. (E) Bebidas diminuídas/excluídas da dieta após diagnóstico da doença. (F) Bebidas aumentadas/incluídas na dieta após diagnóstico da doença. Valores expressos em porcentagem (\%).

Fonte: dados da pesquisa.

No que diz respeito ao recebimento de orientação nutricional por profissional médico e/ou nutricionista, 88,9\% relataram ter recebido (figura 4A). Ao investigar as fontes de informações sobre o comportamento alimentar durante o tratamento do CA, que influenciaram nas respectivas mudanças, seja diminuição/exclusão ou aumento/inclusão de alimentos e/ou bebidas, é possível verificar que, embora majoritariamente tenham recebido informação profissional, a maior influência para as modificações foi mediada através de amigos e/ou familiares e internet (figura 4B). 
Figura 4. Prevalência de orientações nutricionais obtidas por profissionais da saúde, e fontes de informações que influenciaram mudanças comportamentais alimentares após o diagnóstico entre mulheres em tratamento de câncer de mama.
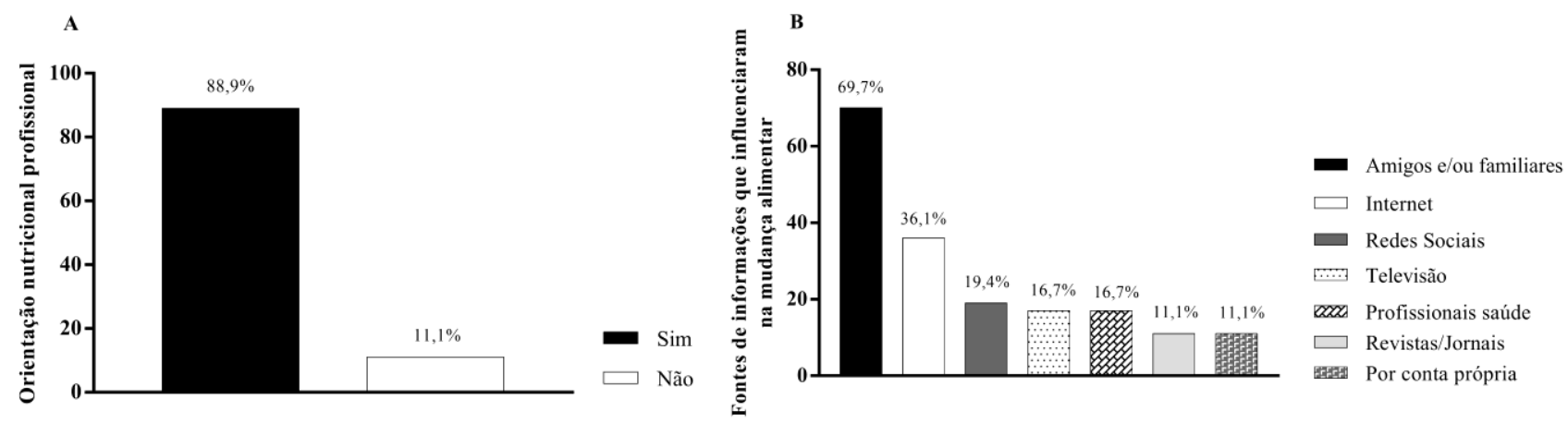

(A) Prevalência de obtenção de orientações nutricionais voltadas ao tratamento do câncer por médico/nutricionista ou não. (B) Fontes de informações que influenciaram as mudanças comportamentais alimentares após o diagnóstico de câncer. Valores expressos em porcentagem $(\%)$.

Fonte: dados da pesquisa.

$\mathrm{Na}$ análise do questionário de frequência alimentar entre os nove diferentes grupos de alimentos, é possível observar que, no que se refere ao grupo de pães, cereais, raízes, massas e tubérculos, as fontes integrais são menos consumidas que os carboidratos simples. O consumo de arroz, macarrão, mandioca, batata, polenta e pães não integrais é consumido diariamente, de uma a duas vezes ao dia pelas que consomem esse tipo de alimento (figura 5A). Entre hortaliças, verduras e legumes, observa-se a prevalência diária no consumo de folhosos crus em majoritariamente uma vez ao dia, seguido pelos vegetais crus e cozidos em menor proporção (figura 5B). Entre as frutas, a banana, o mamão, melão, maçã e manga são frequentemente e em maior quantidade consumidas pelo menos uma vez ao dia, seguida pelo consumo daquelas cítricas. Já o abacate é o fruto menos ingerido, onde 36,1\% nunca o consomem, ou quando consumido é em baixa frequência (figura 5C).

É notório que o consumo de lácteos é feito pelo menos de uma a duas vezes ao dia pela maioria das entrevistadas, mas não há preferência por aqueles do tipo diet ou light, sendo que 83,3\% nunca optam por esses (figura 5D). No grupo das proteínas de origem animal, cabe salientar que a frequência de consumo de aves prevalece, seguido por outros tipos. Metade das mulheres afirmaram que nunca consomem enlatados e embutidos, e dentre as que ingerem, se observa uma baixa frequência no consumo (figura 5E). Destaca-se que a ingesta de leguminosas é prevalente na frequência diária ou de duas a quatro vezes na semana, mas as oleaginosas em menor frequência (figura 5F). Quanto à frequência de consumo de óleos e gorduras, prevalece o consumo diário de óleos vegetais de soja, milho, canola e girassol diariamente, seguido do azeite de oliva. Expressivamente a maioria nunca fez uso de óleo de coco, e evitam a manteiga e a margarina (figura 5G).

Ao avaliar mudanças no comportamento alimentar após o diagnóstico do CA, a maioria das mulheres relatou a redução/exclusão da ingesta de açúcares e doces na dieta, mas quando analisado a frequência de consumo desse grupo, se analisa que o mesmo ainda é majoritariamente consumido diariamente (entre uma a duas vezes ao dia) pelas entrevistadas. A baixa frequência de consumo foi observada entre outros doces, como sobremesas, industrializados e bebidas açucaradas (figura 5H). Entre o grupo classificado como diversos, o café é a bebida mais consumida, sendo ingerida até duas vezes ao dia, seguido pela ingesta de chá. Observam-se uma menor frequência no consumo de bebidas alcoólicas, salgadinhos industrializados, salgadinhos panificados e pizza (figura 5I). 
Figura 5. Frequência alimentar de nove grupos alimentares entre mulheres em tratamento de câncer de mama.
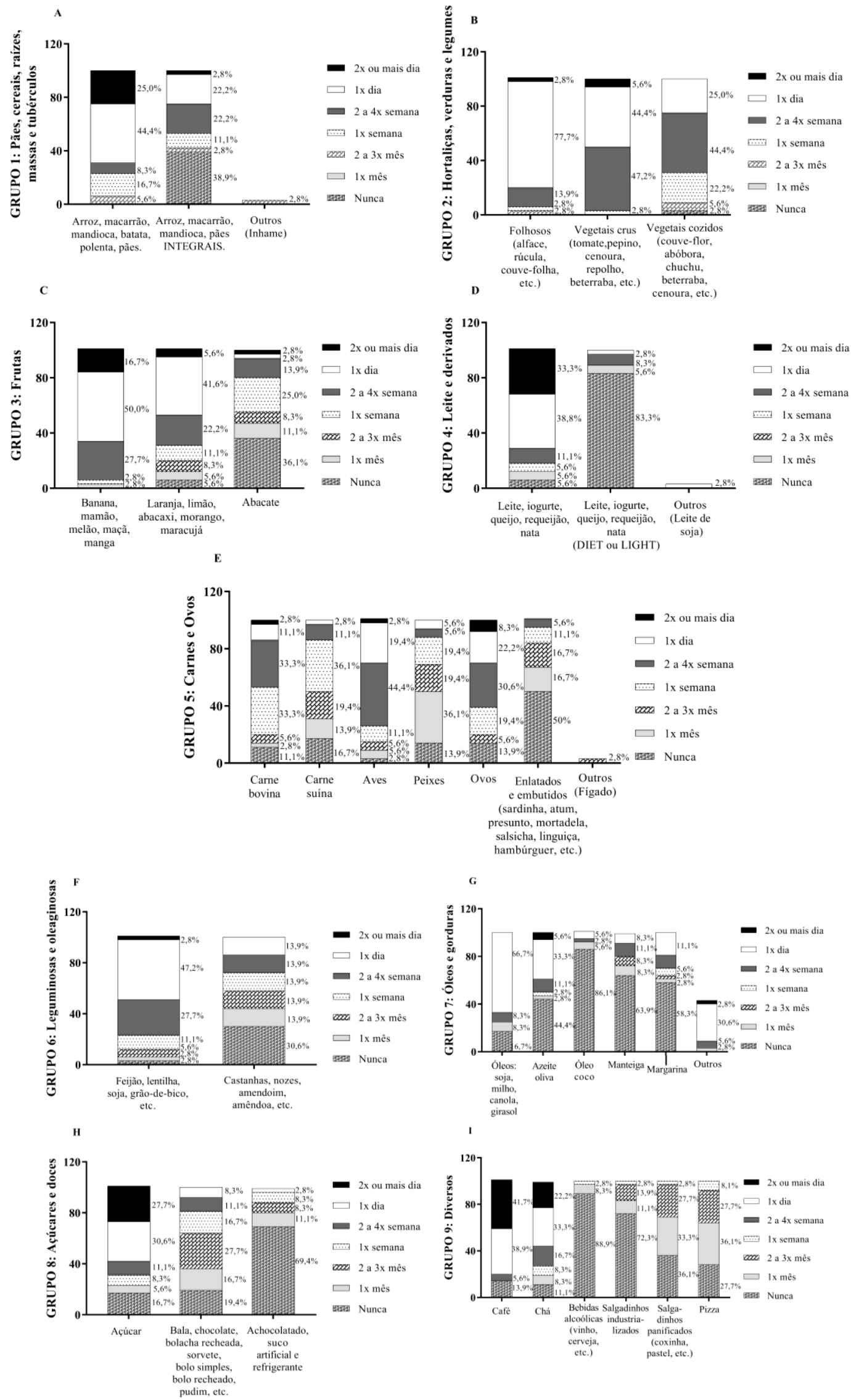
\%: porcentagem. X: vez/vezes. (A) Frequência alimentar do grupo pães, cereais, raízes, massas e tubérculos. (B) Frequência alimentar do grupo hortaliças, verduras e legumes. (C) Frequência alimentar do grupo frutas. (D) Frequência alimentar do grupo leite e derivados. (E) Frequência alimentar do grupo carnes e ovos. (F) Frequência alimentar do grupo leguminosas e oleaginosas. (G) Frequência alimentar do grupo óleos e gorduras. (H) Frequência alimentar do grupo açúcares e doces. (I) Frequência alimentar do grupo diversos. Valores expressos em porcentagem $(\%)$.

Fonte: dados da pesquisa.

\section{Discussão}

Das mulheres avaliadas, a maioria apresentou de baixa a média escolaridade e uma renda de um a dois salários mínimos. De acordo com Shi et al. (2015), melhores condições econômicas estão diretamente relacionadas com a sobrevida, diagnóstico antecipado e menor índice de mortalidade por CA de mama. No estudo de Kameo et al. (2021), ao analisarem 125 pacientes com carcinoma ductal invasivo da mama em tratamento oncológico, constataram que 80,8\% não possuíam ensino superior e 77,6\% tinham renda de um a três salários mínimos.

Predominantemente, as mulheres desse estudo se encontravam acima do peso adequado e com marcadores de risco para doenças metabólicas e cardiovasculares. Resultados também encontrados por Figueiredo et al. (2016), que ao estudarem 50 mulheres com CA de mama em tratamento antineoplásico, evidenciaram o excesso de peso conforme IMC, risco para doenças cardiovasculares e metabólicas considerando a circunferência da cintura. O excesso de peso e a obesidade não são só fatores inerentes ao CA de mama, mas também são fatores preditores da doença (Silva et al., 2019). Para Chan e Norat (2015), a obesidade está associada à resultados negativos do CA de mama. Está vinculada à maior taxa de mortalidade em mulheres que se encontram em pré e/ou pós-menopausa (Bandera \& John, 2018), principalmente em mulheres na pósmenopausa (Silva, 2019).

Para Gomes et al. (2021), em seu estudo, observaram que 64,3\% das mulheres com CA de mama estavam acima do peso, e correlacionaram essa condição à $42,85 \%$ dos casos da doença, sendo que $21,43 \%$ aumentaram de peso após o diagnóstico. No estudo de Almeida et al. (2020), a prevalência do excesso de peso em pacientes com CA de mama foi de 45 a 77,8\%. A obesidade é um dos principais estímulos para o desenvolvimento de CA de mama (Batista et al., 2020). RibeiroSousah et al. (2019), verificaram que o ganho de peso durante o tratamento antineoplásico pode ser causado pelo maior consumo de energia e menor atividade física, visto que, uma maior ingestão alimentar, maior tempo de repouso e redução no nível de atividade física foi observado em mulheres que aumentaram o peso durante o tratamento de CA de mama (n=36), se comparado as que não obtiveram aumento $(n=53)$. É averiguado também que após o início do tratamento, as mulheres estavam em excesso de peso e com a circunferência da cintura aumentada, predispondo às doenças crônicas não-transmissíveis como as cardiovasculares.

Cirurgia, radioterapia, quimioterapia ou a união de ambos pode resultar em dor, náuseas, vômitos, constipação, excesso de peso ou falta de apetite, além de outros efeitos e consequências do tratamento (Palmieri et al., 2013). Predominantemente nesse estudo, as mulheres estavam em tratamento químico e com sintomas mais incidentes de fraqueza/fadiga, seguido de náuseas e inapetência. As modalidades de tratamento do CA de mama podem desencadear o aparecimento de vários efeitos colaterais, como fadiga, náuseas, vômitos, alterações na capacidade intestinal e modificação da percepção do paladar, em consequência disso, mulheres com a doença podem aumentar ou diminuir a ingestão alimentar, vindo a apresentar consumo inadequado (Demark-Wahnefried et al., 2001). Os agentes quimioterápicos possuem a capacidade de intervir na divisão das células, porém, essa funcionalidade não é exclusiva das células neoplásicas, podendo vir a afetar tecidos normais e causar reações adversas, como danos no aparelho gastrointestinal, acarretando inapetência. As lesões causadas no trato gastrointestinal podem facilitar a ocorrência de náuseas e vômitos (Rang et al., 2012).

No estudo de Silva et al. (2010), mulheres em tratamento quimioterápico $(n=21)$ também apresentaram entre os principais efeitos do tratamento fadiga, náuseas e vômitos. Já Atalay e Kuçuk (2015), observaram que o principal efeito 
colateral das 88 mulheres com CA de mama que recebiam quimioterapia foi o ganho de peso. Para Bower et al. (2009), o agravamento da fadiga pode estar diretamente relacionado ao aumento de citocinas inflamatórias durante o tratamento desse tipo de CA, pois observaram que mulheres com esse sintoma possuíam níveis reduzidos de cortisol pela manhã, possivelmente causando alterações no funcionamento do sistema imune, estresse, e presença ou aumento de inflamações.

A alimentação é extremamente importante durante o tratamento do CA, pois favorece o bem-estar dos pacientes, auxiliando na manutenção ou recuperação do estado nutricional, diminuindo os efeitos adversos dos medicamentos. A ação dietética utilizada deve ser individualizada, considerando as necessidades energéticas dos pacientes, suas tolerâncias e aversões alimentares, buscando uma melhor qualidade de vida (Nogueira et al., 2013; INCA, 2016). Detectar precocemente as alterações nutricionais necessárias ao paciente oncológico adulto faz com que a intervenção seja em momento oportuno (INCA). Isso porque, vários problemas nutricionais podem ser desencadeados devido aos efeitos causados pela doença e pela modalidade de tratamento, principalmente se não houver acompanhamento nutricional adequado. É de suma importância o acompanhamento profissional com nutricionista, pois ele poderá orientar a ingestão alimentar de acordo com cada necessidade (Oliveira, 2007).

De acordo com o World Cancer Research Fund (WCRF, 2007), algumas mudanças são fundamentais para prevenir a recidiva da doença e melhorar a qualidade da alimentação, como limitar o consumo de alimentos ricos em açúcar, gordura e sal, moderar o consumo de carne vermelha/processadas e diminuir o consumo de bebidas alcóolicas. Maior consumo de bebidas alcoólicas foi elencado à maior predisposição para de desenvolvimento de CA de mama e, possivelmente, maiores ingestões de fibras dietéticas e frutas associados com o menor risco (Heath et al., 2020). De Liz et al. (2020), elenca que uma intervenção nutricional controlada, com 400 gramas de vegetais por dia e no máximo 500 gramas de carne vermelha/processada por semana estão relacionadas a melhor qualidade alimentar durante o tratamento cirúrgico de CA de mama, melhorando, possivelmente, o prognóstico da doença. Um padrão dietético pró-inflamatório possui influência e aumenta o risco de CA de mama na população feminina (Nogueira, 2019). Dietas vegetarianas parecem ter proteção contra o risco geral de CA (Tantamango-Bartley et al., 2012).

Em sua maioria, as participantes desse estudo, relataram acreditar na influência que a alimentação exerce no tratamento do CA de mama, realizando alterações nos hábitos alimentares. No que tange a esfera da associação de alimentos com incidência e/ou melhora no prognóstico do CA, Rohrmann et al. (2013), associaram que o consumo excessivo de carne processada está ligado não somente à diversos tipos de cânceres, incluindo o de mama, como também às doenças cardiovasculares, e predisposição ao excesso de peso. Lima et al. (2008), atrelaram o consumo de carne vermelha ao CA de mama pelo fato de sua composição nutricional ser rica em gordura saturada, e as carnes processadas por conter potenciais agentes cancerígenos, entre eles a nitrosamina, que eleva o risco de recidiva da doença. Já Cross et al. (2007), afirmaram não haver associação entre o consumo de carne vermelha e CA de mama. No estudo de Sales et al. (2020), foi observado que o consumo de alimentos ultraprocessados representou um terço da alimentação das mulheres sobreviventes ao CA de mama, concomitante à uma redução no consumo de alimentos in natura, fibras e proteínas.

Corroborando com dados de modificações dietéticas desse estudo, ao ser analisado a adesão às recomendações nutricionais preventivas e de recidiva do CA de mama em 201 mulheres em tratamento oncológico, as melhores aderências foram no aumento do consumo de frutas e hortaliças $(73,13 \%)$, limitação no consumo de carnes vermelhas/processadas $(60,70 \%)$ e de bebidas alcoólicas $(88,56 \%)$. Houve diminuição no consumo de bebidas açucaradas $(12,94 \%)$ e aumento de fibras dietéticas (30,35\%). Foi também observado alteração do estado nutricional, com redução do IMC e circunferência da cintura (Palacio et al., 2021). Ambrosi et al. (2011), também observaram aumento no consumo de frutas e vegetais por mulheres em tratamento de CA de mama.

Consumo diminuído ou excluído de refrigerante, ou em uma baixa frequência, foi observado nas mudanças de hábitos 
após diagnóstico do CA entre as mulheres desse estudo, e inversamente proporcional, o chá foi o mais incluído e consumido em maior constância. Chás que contém polifenóis podem ser capazes de inibir a formação de agentes cancerígenos (Hollman \& Arts, 2000). Neste estudo não foram determinados que tipos de chás eram consumidos. Cabe ressaltar, que de acordo com o INCA (2013), os chás não possuem efeitos curativos. No estudo de Nascimento et al. (2019), com 142 participantes, 52,11\% das pacientes com CA de mama consumiam algum fitoterápico ou planta medicinal no formato de chás, ervas ou extratos, em uma frequência de duas vezes por semana a duas vezes ao dia, onde, 43,24\% incluíram esses tipos de produtos após o diagnóstico da doença, com a intenção de reduzir os sintomas apresentados no tratamento ou a massa tumoral, embora, a falta de conhecimento sobre a dosagem adequada de uso tenha sido relatada.

Para desenvolver hábitos alimentares saudáveis é necessário ter conhecimento sobre alimentação. Porém, de forma isolada, o conhecimento individual não é capaz de realizar mudanças no comportamento alimentar, pois pode ser influenciado por vários fatores (Albuquerque et al., 2013). O acompanhamento nutricional durante e após o tratamento para o CA de mama é fundamental, e a orientação nutricional pode ajudar na prevenção de recidivas, já que o IMC diferente do adequado é fator de risco para a doença, e uma alimentação equilibrada e balanceada propicia melhora no peso corporal, e previne o desenvolvimento da doença (Moreira, 2013).

Nesse estudo, a maioria das participantes relatou ter recebido orientação nutricional profissional após o diagnóstico de $\mathrm{CA}$, no entanto, ao investigar as fontes de informações que entusiasmaram as mudanças, os familiares e/ou amigos, internet e redes sociais exerceram maior influência. No estudo de Gomes et al. (2021), 78,57\% das mulheres com CA apresentaram conhecimentos nutricionais moderados. Apesar da condição nutricional apresentar importante papel no tratamento desses pacientes, somente 30 a $60 \%$ recebem terapia nutricional adequada, seja por meio de aconselhamento nutricional, suplementos orais, nutrição enteral ou parenteral (Arends et al., 2017; Planas et al., 2016). Em relação aos conhecimentos nutricionais, no estudo de Rubin (2010), entre mulheres com CA de mama em acompanhamento ambulatorial e não realizando tratamento antineoplásico, foi observado que $61,7 \%$ possuíam conhecimento moderado através da escala de conhecimentos nutricionais. Entre aquelas em tratamento quimioterápico ou radioterápico que não haviam recebido orientações nutricionais profissionais, o conhecimento sobre a interface dieta-doença foi baixo, e a maioria se encontrava com excesso de peso (Sedó, 2013).

Crenças de que certos tipos de alimentos podem estar relacionados à etiopatogenia das neoplasias, e fazer com que os pacientes evitem ou consumam certos grupos alimentares pode fazer com que ocorra um desequilíbrio dietético (Patterson et al., 2003). A população se depara com uma enorme quantidade de informações relacionadas a alimentação e saúde (Cicco et al., 2006). De acordo com o INCA (2019b), há inúmeros boatos sobre CA circulando na internet, desde vacinas à alimentos milagrosos. O gengibre, por exemplo, chegou a ser considerado um agente curativo ao CA de mama, e apesar de que pesquisas vêm sendo realizadas para explicar sua eficácia em ajudar a inibir o crescimento de tumores primários de CA de mama e evitar metástases, nenhum alimento sozinho tem poder de cura (INCA). Um chá chegou a ser indicado no tratamento de CA de mama, porém, apesar de possuir um composto quimiopreventivo chamado acetogenina, não possui poder de cura (Fundação do Câncer, 2019). Pacientes com CA de mama podem se mostrar adeptos a quaisquer opções de tratamento para atingir a cura ou atenuar o seu sofrimento (Nascimento, 2019).

\section{Conclusão}

Diante do analisado, observou-se que a média entre as mulheres é de um público que apresenta sobrepeso, risco para complicações metabólicas muito elevado e risco para doenças cardiovasculares. Apenas a circunferência do braço classificou como eutrófica a maioria das entrevistadas.

Os sintomas como fraqueza, náuseas e inapetência foram os mais relatados durante o tratamento, e podem ser atrelados a terapia oncológica por quimioterápicos realizado pela maioria das mulheres no período de coleta dos dados. Esses 
sintomas impactam diretamente sobre a via alimentar e, consequentemente, estado nutricional e perspectiva alimentar durante o tratamento.

Evidencia-se que mesmo valorizando a importância da alimentação e do profissional de saúde no tratamento do CA, as modificações de hábitos, seja por aumento ou diminuição, inclusão ou exclusão de alimentos e/ou bebidas, foi por influência preferencialmente de amigos e/ou familiares, internet e redes sociais. Essa constatação levanta a importância dos profissionais de saúde se apropriarem desse saber, e se manterem ativos tanto no desenvolvimento de técnicas, quanto na forma eficaz e constante de repasse de informações corretas sobre o assunto.

Após o diagnóstico, é notório que houve mudanças na conduta alimentar, principalmente pela diminuição e/ou exclusão de alimentos açucarados/doces, gorduras, carnes vermelhas, refrigerantes, e bebidas alcoólicas; e inclusão de frutas, hortaliças, chás, sucos naturais, e água. Na frequência alimentar, percebe-se que o consumo de carboidratos simples é superior aos complexos; mais hortaliças folhosas e vegetais crus frente aos cozidos; maior consumo de frutas adocicadas em detrimento as cítricas e abacate; mais lácteos integrais que aqueles diet e light; maior constância de consumo frango como proteína animal, e leguminosas entre as de origem vegetal. Maior frequência de consumo de óleos vegetais, azeite de oliva e banha de porco que outras gorduras. Percebe-se ainda, uma redução no consumo de embutidos, industrializados, e alimentos doces como sobremesas, bolos, entre outros. Porém, embora relato da diminuição na quantidade, nota-se o consumo frequente de açúcar em até duas vezes ao dia.

Pesquisas dessa grandeza, que tendem a entender o impacto de uma doença orgânica ou de seu tratamento sobre o estado de saúde, com ênfase no estado nutricional, amparando e investigando o comportamento, influências e percepções alimentares após o diagnóstico de CA, são de extrema importância e relevância para a prática clínica e científica, pois é por meio desses dados que é analisado e traçado novos amparos de suporte multiprofissional para a fidelização de um tratamento satisfatório e menos danoso para o organismo.

Sugere-se para trabalhos futuros a averiguação da relação entre peso e circunferências associadas ao tipo de tratamento; alimentos antioxidantes ou com outras propriedades benéficas para a recuperação da saúde e relação com efeitos colaterais em quimioterapia, radioterapia e/ou pós-cirurgia; bem como, determinar os tipos de chás consumidos e sua possível interação com a doença e/ou com o tratamento, visto o aumento do consumo desse após o diagnóstico e/ou tratamento oncológico. Ainda, é necessário apurar a influência dos alimentos de forma isolada e de padrões alimentares sobre a influência no desenvolvimento e tratamento do CA de mama, e como isso impacta no comportamento, perspectiva e influências sofridas por essas pacientes.

\section{Referências}

Albuquerque, L. S., Carneiro, P. C. P. D. M., Oliveira, M. R., \& Verde, S. M. M. L. (2013). Influência da educação nutricional no conhecimento sobre a relação dieta-doença de mulheres mastectomizadas. Revista da Sociedade Brasileira de Alimentação e Nutrição, 38(2), 97-114. http://dx.doi.org/10.4322/nutrire.2013.011

Almeida, A. B. M. S., Almeida, A. V. V., \& Silva, L. D. S. (2020). Prevalência de excesso de peso em pacientes com câncer de mama submetidas ao tratamento quimioterápico [Trabalho de Conclusão de Curso, Centro Universitário Tiradentes]. https://openrit.grupotiradentes.com/xmlui/handle/set/3261

Ambrosi, C. Di Pietro, P. F., Rockenbach, G., Vieira, F. G. K., Galvan, D., Crippa, C. G., \& Fausto, M. A. (2011). Fatores que influenciam o consumo energético de mulheres no tratamento do câncer de mama. Revista Brasileia de Ginecologia e Obstetrícia, 33(8), 207-213. https://doi.org/10.1590/S010072032011000800007

Arends, J., Baracos, V., Bertz, H., Bozzetti, F., Calder, P. C., Deutz, N. E. P., Erickson, A., Laviano, M., Lisanti, D. N., Lobo, D., McMillan, M., Muscaritoli, J., Ockenga, M., Pirlich, F. Strasser, M. de van der Schuren, A. Van Gossum, P. \& Weimann, A. (2017). ESPEN expert group recommendations for action against cancer-related malnutrition. Clinical Nutrition, 36(5), 1187-1196. https://doi.org/10.1016/j.clnu.2017.06.017

Atalay C., \& Kuçuk A. (2015). The impact of weight gain during adjuvante chemotherapy on survival in breast câncer. Turkish Journal of Surgery, 31(3), 124127. 10.5152/UCD.2015.3123

Bandera, E. V., \& John, E. M. (2018). Obesity, Body Composition, and Breast Cancer. JAMA Oncology, 4(6), 804. https://doi.org/10.1001/jamaoncol.2018.0125 
Batista, G. V., Moreira, J. A., Leite, A. L. \& Moreira, C. I. H. (2020). Câncer de mama: fatores de risco e métodos de prevenção. Research, Society e Development, 9(12). http://dx.doi.org/10.33448/rsd-v9i12.11077

Bower, J. E., Ganz, P. A., Tao, M. L., Hu, W., Belin, T. R., Sepah, S., Cole, S., Aziz, N. (2009). Inflammatory Biomarkers and Fatigue during Radiation Therapy for Breast and Prostate Cancer. Clinical Cancer Research, 15(17), 5534-5540. 10.1158/1078-0432.ccr-08-2584

Chan, D. S. M., \& Norat, T. (2015). Obesity and Breast Cancer: Not Only a Risk Factor of the Disease. Current Treatment Options in Oncology. 16(5). $10.1007 / \mathrm{s} 11864-015-0341-9$

Cicco, M. F., Santos, N. O., Silva, M. M., Laham, C., Junior, A. G., \& Lucia, M. C. S. (2006). Imagem corporal, práticas de dietas e crenças alimentares em adolescentes e adultas. Psicologia Hospitalar, 4(1), 01-27. http://pepsic.bvsalud.org/scielo.php?script=sci_abstract\&pid=S1677-

$74092006000100002 \& \operatorname{lng}=\mathrm{pt} \& n r m=i \& t \operatorname{lng}=\mathrm{pt}$

Cross, A. J., Leitzmann, M. F., Gail, M. H., Hollenbeck, A. R., Schatzkin, A., \& Sinha, R. (2007). A Prospective Study of Red and Processed Meat Intake in Relation to Cancer Risk. PLoS Medicine, 4(12). https://doi.org/10.1371/journal.pmed.0040325

Cuppari, L. (2005). Guia de nutrição: nutrição clínica no adulto (2a ed). Barueri: Manole.

De Liz, S., Vieira, F. G. K., Geraldo, A. P. G., Assis, M. A. A., \& Di Pietro, P. F. (2020). Educational nutritional intervention program improved the quality of diet of women with breast cancer in adjuvant treatment. Revista de Nutrição, 33. https://doi.org/10.1590/1678-9865202033e190145

Demark-Wahnefried, W., Peterson, B. L., Winer, E. P., Marks, L., Aziz, N., Marcom, P. K., Blackwell, K., \& Rimer, B. K. (2001). Changes in Weight, Body Composition, and Factors Influencing Energy Balance Among Premenopausal Breast Cancer Patients Receiving Adjuvant Chemotherapy. Journal of Clinical Oncology, 19(9), 2381-2389. https://doi.org/10.1200/JCO.2001.19.9.2381

Figueiredo, A. C. D. S., Ferreira, R. N. F., Duarte, M. A. G., Coelho, A. F., \& Cabral, K. M. A. A. (2016). Prevalência da obesidade em mulheres tratadas de câncer de mama numa UNACOM em Juiz de Fora. Revista Brasileira de Mastologia, 26(4), 169-174. https://doi.org/10.5327/Z201600040006RBM

Fonseca, J. J. S. (2002). Metodologia da pesquisa científica. Fortaleza: UEC.

Frisancho, A. R. (1981). New norms of upper limb fat and muscle areas for assessment of nutritional status. American Journal of Clinical Nutrition, 34(11), 2540-2545. http://dx.doi.org/10.1093/ajcn/34.11.2540

Fundação do Câncer. (2012, 24 Abril). O chá de graviola é indicado com o tratamento do câncer?. https://www.cancer.org.br/faqs/o-cha-de-graviola-eindicado-com-o-tratamento-do-cancer/.

Gomes, G. C. S., Ferreira, C. G., Pereira, I. M., Cristovão, T. C. S., \& Genaro, S. C. (2021). Conhecimento alimentar e perfil antropométrico de mulheres com câncer de mama em tratamento quimioterápico. Brazilian Journal of Development. 7(7), 73851-73870. doi: 10.34117/bjdv7n7-522

Heath, A. K., Muller, D. C., van den Brandt, P. A., Papadimitriou, N., Critselis, E., Gunter, M., ... Boeing, H. (2020). Nutrient-wide association study of 92 foods and nutrients and breast cancer risk. Breast Cancer Research, 22(1). https://doi.org/10.34117/bjdv7n7-522

Hollman, P. C., \& Arts, I. C. (2000). Flavonols, flavones and flavanols - nature, occurrence and dietary burden. Journal of the Science of Food and Agriculture, 80(7), 1081-1093. https://doi.org/10.1002/(SICI)1097-0010(20000515)80:7<1081::AID-JSFA566>3.0.CO;2-G

Instituto Nacional do Câncer José Alencar Gomes da Silva. (2013). Tratamentos alternativos: mitos e verdades. Rio de Janeiro. https://issuu.com/sbcom/docs/rc20_baixa

Instituto Nacional de Câncer José Alencar Gomes da Silva. (2016). Consenso Nacional nutrição oncológica (2a ed., vol. 2). Rio de Janeiro. https://www.inca.gov.br/sites/ufu.sti.inca.local/files//media/document//consenso-nutricao-oncologica-vol-ii-2-ed-2016.pdf

Instituto Nacional de Câncer José Alencar Gomes da Silva. (2019a). Estimativa 2020: incidência de Câncer no Brasil. Rio de Ja neiro. https://www.inca.gov.br/sites/ufu.sti.inca.local/files//media/document//estimativa-2020-incidencia-de-cancer-no-brasil.pdf

Instituto Nacional de Câncer José Alencar Gomes da Silva. (2019b, 08 Fevereiro). Notícias falsas podem prejudicar diagnóstico e tratamento do câncer. https://www.inca.gov.br/en/node/3108.

Instituto Nacional de Câncer José Alencar Gomes da Silva. (2019c). A situação do câncer de mama no Brasil: Síntese de dados dos sistemas de informação. Rio de Janeiro. https://www.inca.gov.br/sites/ufu.sti.inca.local/files//media/document//a_situacao_ca_mama_brasil_2019.pdf.

Instituto Nacional do Câncer José Alencar Gomes da Silva. (2020a). ABC do câncer: abordagens básicas para o controle do câncer (6a ed.). Rio de Janeiro. https://www.inca.gov.br/sites/ufu.sti.inca.local/files//media/document//livro-abc-6-edicao-2020.pdf

Instituto Nacional do Câncer José Alencar Gomes da Silva. (2020b, 02 Setembro). Tipos de câncer: câncer de mama. https://www.inca.gov.br/tipos-decancer/cancer-de-mama

Kameo, S. Y., Lima, R. B., Ramos, M. J. O., Fonseca, T. V., Vassilievitch, A. C., Costa, J. S.. Santos, J. C. O., Santos, D. K. C., Amorim, B. F., Marinho, P. M. L., Sawada, N. O., \& Silva, G. M. (2021). Perfil clínico-epidemiológico de mulheres em tratamento oncológico de carcinoma ductal invasivo da mama. Research, Society e Development. 10(1). http://dx.doi.org/10.33448/rsd-v10i1.11836

Lima, F. E. L. de, Latorre, M. do R. D. de O., Costa, M. J. de C., \& Fisberg, R. M. (2008). Diet and cancer in Northeast Brazil: evaluation of eating habits and food group consumption in relation to breast cancer. Cadernos de Saúde Pública, 24(4), 820-828. https://doi.org/10.1590/S0102-311X2008000400012

Mahan, L. K., \& Raymond, J. L. (2018). Krause: alimentos, nutrição e dietoterapia (14a ed). Rio de Janeiro: Elsevier. 
Moreira, L. F. C. (2013). Impacto do Tratamento Quimioterápico no Estado Nutricional e no Comportamento Alimentar de Pacientes com Neoplasia Mamária [Trabalho de conclusão de curso, Centro Universitário de Brasília]. https://repositorio.uniceub.br/jspui/bitstream/235/3942/1/monog1.pdf

Nascimento, B. P., Machado, T. J. S., \& Aragão, k. s. (2019). Análise do uso de fitoterápicos e plantas medicinais por pacientes com câncer de mama. Biosaúde, 21(9). https://www.uel.br/revistas/uel/index.php/biosaude/article/view/38847/27560

Nogueira, D., Justi J., \& Freire, H. B. G. (2013). Correlação entre imagem corporal, mastectomia e aumento de peso: um estudo de caso. Revista Interdisciplinar de Estudos em Saúde, 2(2), 25-36. https://doi.org/10.33362/ries.v2i2.152

Nogueira, T. R., Caldas, D. R. C., Araújo, C. G. B., Silva, M. C. M., Nogueira, N. N., Rodrigues, G. P. (2019). Potencial inflamatório da dieta e risco de câncer de mama. Revista Eletrônica Acervo de Saúde, 22. https://doi.org/10.25248/reas.e571.2019

Oliveira, T. (2007). A importância do acompanhamento nutricional para pacientes com câncer. Prática Hospitalar, 51. http://nu triliv.com.br/wpcontent/uploads/2012/02/A-importanciadoAcompanhamento-Nuticional-para-Pacientes-com-cancer.pdf

Palacio, B. Q. E., Sampaio, H. A. C., Henriques, E. M. V., Carioca, A. A. F., \& Vergara, C. M. A. C. (2021). Adesão de sobreviventes de câncer de mama às recomendações nutricionais preventivas da recidiva da doença. Interação, 21(2), 11-27. 10.53660/inter-90-s108-p11-27

Palmieri, B. N., Moulatlet, E. M., Buschinelli, L. K. O., \& Pinto-e-Silva, M. E. M. (2013). Aceitação de preparações e sua associação com os sintomas decorrentes do tratamento de câncer em pacientes de uma clínica especializada. Cadernos Saúde Coletiva, 21(1), 02-09. http://dx.doi.org/10.1590/S1414$462 \times 2013000100002$

Patterson, R., Neuhouser, M., Hedderson, M., Schwartz, S., Standish, L., \& Bowen, D. (2003). Changes in diet, physical activity, and supplement use among adults diagnosed with cancer. Journal of the American Dietetic Association, 103(3), 323-328. https://doi.org/10.1053/jada.2003.50045

Pereira, A. S., Shitsuka, D. M., Pereira, F. J., \& Shitsuka, R. (2018). Metodologia da pesquisa científica. Santa Maria: UFSM. https://repositorio.ufsm.br/bitstream/handle/1/15824/Lic_Computacao_Metodologia-Pesquisa-Cientifica.pdf?sequence=1

Planas, M., Álvarez-Hernández, J., León-Sanz, M., Celaya-Pérez, S., Araujo, K., \& García de Lorenzo, A. (2016). Prevalence of hospital malnutrition in cancer patients: a sub-analysis of the PREDyCES® study. Supportive Care in Cancer, 24(1), 429-435. https://doi.org/10.1007/s00520-015-2813-7

Rang, H. P., Dale, M. M., Ritter, J. J., Flower R. J., \& Henderson, G. (2012). Rang \& Dale: farmacologia (7a ed). Rio de Janeiro: Elsevier.

Ribeiro-Sousah, M. A. S., Mastelaro, I., Peria, F. M. Carrara, H. A., Andrade, J. M., \& Cunha, S. F. C. C. (2019). Weight gain during systemic oncologic therapy for breast câncer: changes in food intake and physical activity. Revista Brasileira de Cancerologia, 65(2). https://doi.org/10.32635/21769745.RBC.2019v65n2.360

Rohrmann, S., Overvad, K., Bueno-de-Mesquita, H. B., Jakobsen, M. U., Egeberg, R., Tjønneland, A., ... Linseisen, J. (2013). Meat consumption and mortality - results from the European Prospective Investigation into Cancer and Nutrition. BMC Medicine, 11(1). doi:10.1186/1741-7015-11-63

Rubin, B. A., Airton, T. S., Zelmanowics, A. M., \& Rosa, D. D. (2010). Perfil Antropométrico e Conhecimento Nutricional de Mulheres Sobreviventes de Câncer de Mama do Sul do Brasil. Antropometria e Conhecimento Nutricional, 56(3), 303-309. https://rbc.inca.gov.br/revista/index.php/revista/article/view/1478/867

Sales, J. N., Manuella, C. B., Bezerra, I. N., \& Verde, S. M. M. (2020). Potencial infla matório da dieta e risco de câncer de mama. Revista Eletrônica Acervo de Saúde. 66(3). https://doi.org/10.25248/reas.e571.2019

Sawaya, A. L., Leandro, C. G., \& Waitzberg, D. L. (2018). Fisiologia da nutrição na saúde e na doença: da biologia molecular ao tratamento (2a ed). Rio de Janeiro: Atheneu.

Sedó, K. S., Lima, C. A., Carneiro, P. C. P. D., Alburquerque, L. S., Araújo, C. O., Castro, A. S., \& Verde, S. M. M. L. (2013). Nutritional knowledge of women with breast cancer and its relationship with nutritional status. Brazilian Journal in Health Promotion, 26(1), 71-78. http://dx.doi.org/10.5020/2626

Shi, R., Taylor, H., McLarty, J., Liu, L., Mills, G., \& Burton, G. (2015). Effects of payer status on breast cancer survival: a retrospective study. BMC Cancer, 15(1). https://doi.org/10.1186/s12885-015-1228-7

Silva C. B., Alburquerque, V., \& Leite, J. (2010). Qualidade de vida em pacientes portadoras de neoplasia mamária submetidas a tratamento quimioterápico. Revista Brasileira de Cancerologia. 56(2), 227-236. https://rbc.inca.gov.br/revista/index.php/revista/article/view/1501/902

Silva, H. R., Costa, R. H. F., Neto, J. C. P., Júnior, C. A. A. M. Pacheco, N. I., Brito, N. S., Soares, F. C. S., Sousa, L. K. R., Reis, J. A. S., Oliveira, J. E. A., \& Pessoa, G. T. (2019). Associação prevalência e fatores de risco entre obesidade e câncer de mama. Research, Society e Development. 9(3). http://dx.doi.org/10.33448/rsd-v9i3.2385

Tantamango-Bartley, Y., Jaceldo-Siegl, K., Fan, J., \& Fraser, G. (2012). Vegetarian Diets and the Incidence of Cancer in a Low-risk Population. Cancer Epidemiology Biomarkers \& Prevention, 22(2), 286-294. doi:10.1158/1055-9965.epi-12-1060

Triviños, A. N. S. (1987). Introdução à pesquisa em ciências sociais: a pesquisa qualitativa em educação. São Paulo: Atlas.

Viebig, R. F., \& Valero, M. P. (2004). Desenvolvimento de um questionário de frequência alimentar para o estudo de doenças não transmissíveis. Revista de Saúde Pública, 38(4), 581-584. https://doi.org/10.1590/S0034-89102004000400016

World Cancer Research Fund. (2007). Food, Nutrition, Physical Activity, and the Prevention of Cancer: a Global Perspective. Washington DC. https://www.wcrf.org/dietandcancer/a-summary-of-the-third-expert-report/

World Health Organization. (1997). Obesity: preventing and managing the global epidemic. Geneva. https://apps.who.int/iris/handle/10665/63854 